\title{
Finite element modeling of nearly incompressible bonds
}

\author{
Gregory J. Michels*, Victor L. Genberg \\ Sigmadyne, Inc. \\ 803 West Avenue, Rochester, NY 14611 \\ Keith B. Doyle \\ Optical Research Associates \\ 1800 West Park Drive, Westborough, MA
}

\begin{abstract}
Proper finite element modeling of nearly incompressible bonds which are commonly used to mount optics requires special considerations. Modeling difficulties arise due to the geometries of the mounting designs and the incompressible nature of the bonding materials involved in many applications. Therefore, specific methods of modeling must be employed. Such methods of modeling two common bond designs are outlined. Treatment of these modeling methods includes why they are needed, how they are performed, and what their limitations are.
\end{abstract}

Keywords: Finite element analysis, bonds, opto-mechanics, mounts, RTV, incompressible

\section{INTRODUCTION}

Finite element modeling of nearly incompressible bonds must be performed with caution in order to represent the elastic stiffness correctly. Treatment of the effective modulus through-the-thickness of thin nearly incompressible bonds has been the topic of several sources in the published literature. ${ }^{1,2,3}$ However, these sources have two principal limitations. None give a complete description of how the full elastic description of Hooke's law should be represented by effective properties for coarse bond models. Furthermore, no practical methods are presented by which one can obtain effective properties for various bond geometries. The work presented in this paper seeks to familiarize the reader with readily useable stiffness characterizations for some common bond geometries and a general method by which stiffness representations may be developed for specialized bond designs.

\section{IMPORTANT PROPERTIES OF NEARLY INCOMPRESSIBLE MATERIALS}

Before presenting the methods by which nearly incompressible bonds can be modeled, it is imperative to familiarize the reader with some relevant characteristics of incompressible bonds which must be included in a finite element model in order to obtain an accurate stiffness representation. Equation (1) is the three dimensional form of the stress vs. strain relationship for linear elastic isotropic materials which is also known as Hooke's law.

\footnotetext{
* Correspondence: Email: michels@ sigmadyne.com; www: http://www.sigmadyne.com; Telephone: (585) 235-6892
} 


$$
\left\{\begin{array}{l}
\sigma_{11} \\
\sigma_{22} \\
\sigma_{33} \\
\tau_{12} \\
\tau_{23} \\
\tau_{31}
\end{array}\right\}=\left[\begin{array}{cccccc}
\frac{(1-v) E}{(1+v)(1-2 v)} & \frac{v E}{(1+v)(1-2 v)} & \frac{v E}{(1+v)(1-2 v)} & 0 & 0 & 0 \\
\frac{v E}{(1+v)(1-2 v)} & \frac{(1-v) E}{(1+v)(1-2 v)} & \frac{v E}{(1+v)(1-2 v)} & 0 & 0 & 0 \\
\frac{v E}{(1+v)(1-2 v)} & \frac{v E}{(1+v)(1-2 v)} & \frac{(1-v) E}{(1+v)(1-2 v)} & 0 & 0 & 0 \\
0 & 0 & 0 & G & 0 & 0 \\
0 & 0 & 0 & 0 & G & 0 \\
0 & 0 & 0 & 0 & 0 & G
\end{array}\right\}\left\{\begin{array}{l}
\varepsilon_{11} \\
\varepsilon_{22} \\
\varepsilon_{33} \\
\gamma_{12} \\
\gamma_{23} \\
\gamma_{31}
\end{array}\right\}
$$

Figures 1(a) and 1(b) show a uniaxial and a thin layer tension test sample, respectively, which are loaded by rigid substrates as shown. The uniaxial tension test sample is characterized by a very low diameter to thickness ratio, $D / t$, while the thin layer tension test sample is characterized by a very high $D / t$ ratio. If a very long uniaxial tension test sample is stressed an amount $\sigma$ and a resulting strain $\varepsilon$ is measured, then the ratio of the stress to the strain will be the Young's modulus, $E$. We can see this by substituting $\varepsilon_{11}=-v \varepsilon, \varepsilon_{22}=-v \varepsilon, \varepsilon_{33}=\varepsilon$, and $\sigma_{33}=\sigma$ into the expression for $\sigma_{33}$ in Eqn. (1) and simplifying.

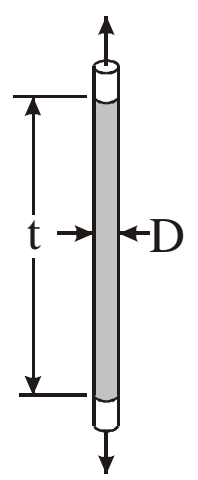

(a)

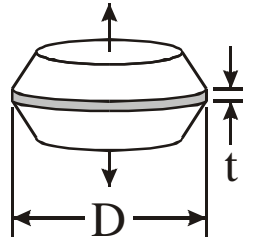

(b)
Figure 1: Two extremes of tension test samples; (a) uniaxial tension test sample and (b) thin layer tension test sample.

$$
\sigma=\frac{v E}{(1+v)(1-2 v)}(-v \varepsilon)+\frac{v E}{(1+v)(1-2 v)}(-v \varepsilon)+\frac{(1-v) E}{(1+v)(1-2 v)}(\varepsilon)=\frac{\left(1-v-2 v^{2}\right) \varepsilon E}{(1+v)(1-2 v)}=\varepsilon E
$$

Dividing both sides of the equation by $\varepsilon$ shows that the $\sigma / \varepsilon$ ratio of a sufficiently long tensile test sample is the Young's modulus, $E$.

$$
\frac{\sigma}{\varepsilon}=E
$$

But for a thin layer tension test sample with a very high $D / t$ ratio we make the assumption that $\varepsilon_{11}=0, \varepsilon_{22}=0, \varepsilon_{33}=\varepsilon$, and $\sigma_{33}=\sigma$ into the expression for $\sigma_{33}$ in Eqn. (1). The result is given in Eqn. (4).

$$
\frac{\sigma}{\varepsilon}=\frac{(1-v) E}{(1+v)(1-2 v)}=m E=M
$$

Equation (4) shows that a thin layer tension test sample acts with a very different stress vs. strain relationship than was seen for the uniaxial test sample. The $\sigma / \varepsilon$ ratio for the thin layer sample is called the maximum modulus, $M$. The maximum modulus is the upper bound on the stress to strain ratio of thin layer samples for increasing diameter to thickness ratios. Tension samples with $D / t$ ratios between these two extremes display stress to strain ratios which fall between the Young's modulus and the maximum modulus.

Figure 2 illustrates the behavior of such a spectrum of test samples with curves of $\sigma / \varepsilon$ vs. $D / t$ normalized by $E$. A family of curves are plotted for various values of Poisson's ratio. One important observation to be made from Figure 2 is that $\sigma / \varepsilon$ is dependent on the geometry of the test sample for high Poisson ratios. That is, samples with low $D / t$ ratios have $\sigma / \varepsilon$ ratios near

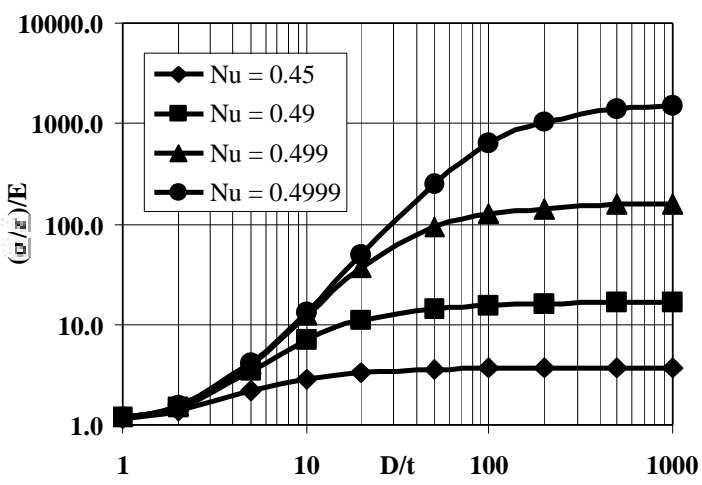

Figure 2: Plot of $(\sigma / \varepsilon) / E$ vs. $D / t$ showing effect of $D / t$ ratio and Poisson's ratio on tension test sample stiffness. 
$E$ but display higher $\sigma / \varepsilon$ ratios with increasing $D / t$ ratios. Samples with greater $D / t$ ratios display greater $\sigma / \varepsilon$ ratios due to an increased resistance to the Poisson effect. The Poisson effect becomes more localized at the edges of the sample in cases where the $D / t$ ratio is greater. Therefore, it is important when constructing a finite element model to allow the bond model the ability to predict such deformation in order to obtain an accurate representation of the bond stiffness.

A second important observation to be made from Figure 2 is that the $\sigma / \varepsilon$ ratio of the test sample is a strong function of Poisson's ratio for samples with high $D / t$ ratios. Notice from Figure 2 that the maximum modulus increases by an order of magnitude with every "9" which is added to the value of Poisson's ratio. Therefore, when modeling nearly incompressible bonds it is very important that the proper value of Poisson's ratio be included.

\section{MODELING METHODS}

There are basically two approaches to modeling nearly incompressible bonds. The difference between each approach is in how the volume preserving deformations are represented in each model type. However, each method utilizes the Young's modulus, $E$, and bulk modulus, $B$, obtained from tests. Poisson's ratio can then be calculated from the Young's modulus and bulk modulus.

$$
v=\frac{1}{2}-\frac{E}{6 B}
$$

The shear modulus can then be computed as,

$$
G=\frac{E}{2(1+v)} .
$$

\subsection{Detailed model}

The most obvious and straightforward method of including the volume preserving deformations is to use enough detail in a solid finite element mesh of the bond to allow their prediction in the analysis. In such a model the properties discussed above are referenced on the material descriptions for the bonding material. Figure 3 shows an example of such a model.

Usually the use of four tri-linear solid elements on the free surfaces of the bond is sufficient to predict the volume preserving deformations. However, there must also be sufficient fidelity to represent the decay in such deformations with distance from the free faces.

\subsection{Effective model}

One problem with the detailed model described above is that it requires mesh fidelity which exceeds the desired mesh fidelity of the surrounding structure. This makes integration of the bond mesh into the adjacent meshes very difficult without adding unwanted fidelity to a significant portion of the model as illustrated in Figure 3. In addition, since typical bond thicknesses can be very small, using several elements through the thickness can generate large aspect ratios in the elements used to mesh the bond. Therefore, it is desirable to develop a method by which a coarse bond model may

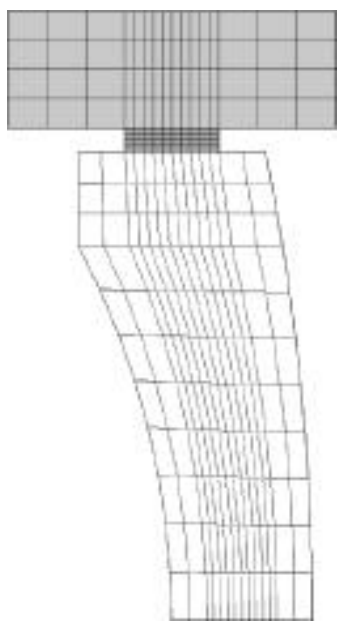

Figure 3: Cross-section plot of detailed ring bond model, lens, and barrel. be used with only one element through the thickness.

To begin development of such a method we must first understand that using only one element through the thickness of a thin layer tension test sample enforces the strains in the plane of the solid mesh to be zero. As was demonstrated above, this causes the ratio of the through-the-thickness stress to strain ratio to be the maximum modulus. However, if the bond has some finite $D / t$ ratio, the real hardware will not act with such excessive stiffness because compliance will be 
introduced due to volume preserving deformation at the free surface. In order to correct such a situation effective material properties can be employed for the simplified bond mesh so that it will behave with the correct average stiffness. Such effective properties can be obtained for a particular bond design and bond material by running some simple finite element test cases. The goal of these test cases is to interrogate a detailed model of the bond to determine an effective Hooke's law matrix which replaces that given in Eqn. (1) and is to be used with coarse bond models. The form of the effective property is given as,

$$
\left\{\begin{array}{l}
\sigma_{11} \\
\sigma_{22} \\
\sigma_{33} \\
\tau_{12} \\
\tau_{23} \\
\tau_{31}
\end{array}\right\}=\left[\begin{array}{cccccc}
k_{11} M & \frac{k_{12} k_{11} v M}{(1-v)} & \frac{k_{13} k_{11} v M}{(1-v)} & 0 & 0 & 0 \\
\frac{k_{21} k_{22} v M}{(1-v)} & k_{22} M & \frac{k_{23} k_{22} v M}{(1-v)} & 0 & 0 & 0 \\
\frac{k_{31} k_{33} v M}{(1-v)} & \frac{k_{32} k_{33} v M}{(1-v)} & k_{33} M & 0 & 0 & 0 \\
0 & 0 & 0 & G & 0 & 0 \\
0 & 0 & 0 & 0 & G & 0 \\
0 & 0 & 0 & 0 & 0 & G
\end{array}\right]\left\{\begin{array}{c}
\varepsilon_{11} \\
\varepsilon_{22} \\
\varepsilon_{33} \\
\gamma_{12} \\
\gamma_{23} \\
\gamma_{31}
\end{array}\right\},
$$

where, the correction factors, $k_{i j}$, are given by,

$$
k_{i i}=\frac{\sum_{i i}}{M}, \quad k_{i j}=\frac{(1-v) \Sigma_{i j}}{v k_{i i} M} \quad i \neq j,
$$

$\Sigma_{i i}$ is the stress in the $i^{\text {th }}$ direction due to a unit strain in the $i^{\text {th }}$ direction with all other strains are zero, and $\Sigma_{i j}$ is the stress in the $i^{\text {th }}$ direction due to a unit strain in the $j^{\text {th }}$ direction with all other strains zero.

The method for developing this stress vs. strain relationship is best illustrated by example.

\subsubsection{Development of effective properties for a "hockey puck" bond}

Figure 4 shows an example of a "hockey puck" bond for which effective properties are desired. It is defined that the " 3 " direction is through the thickness of the bond while the " 1 " and " 2 " directions are the radial and hoop directions, respectively, in the plane of the bond. A wedge of the full bond pad was modeled in MSC/NASTRAN ${ }^{\top M}$ with sufficient detail to allow prediction of the volume preserving deformation of the bond. This test case model is shown in Figure 5. Symmetric boundary conditions were applied to the model to mimic the rest of the bond, and additional constraints in the radial direction were applied to the top and bottom surfaces to simulate rigid substrates.

Two enforced displacement analysis cases were then performed which each generate unit strains of the bond. In the first load case all nodes on the top surface were given an enforced vertical displacement equal to the thickness of the bond. This deformation prescription generates a unit strain in the through-thethickness direction while the overall strains in the radial and hoop directions were constrained to zero. The net force required to generate this deformation, $F_{33}$, was then used with Eqn. (8) to compute $k_{33}$ as,

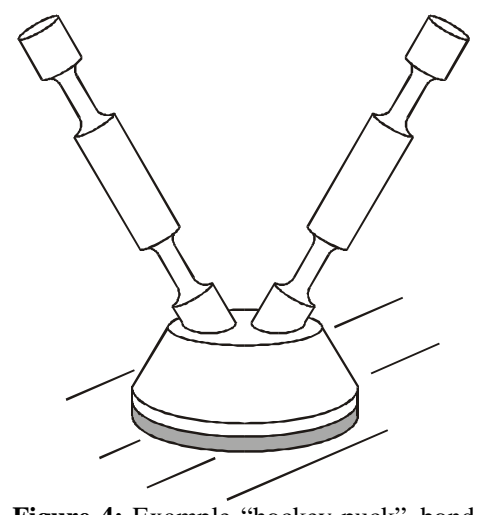

Figure 4: Example "hockey puck" bond design.
Figure 5: Test case model used to generate effective properties for "hockey puck" bonds. 


$$
k_{33}=\frac{F_{33}}{A M},
$$

where, $A$ is the area of the top surface of the wedge model.

The product of $k_{33}$ and $M$ has been commonly referred to in the literature as $E_{c}$. However, as indicated by Eqns. (7) and (8), this quantity alone is insufficient to fully define the material behavior. The quantity $k_{33} M$ only defines the ratio of the through-the-thickness stress to the through-the-thickness strain assuming the lateral strains are zero. The off-diagonal terms shown in Eqn. (7) are also needed to define how orthogonal strains are coupled to one another. For example, the thermoelastic growth of the substrates of a "hockey puck" bond will cause radial and hoop strains in the bond. Due to the Poisson effect the bond will undergo a resulting strain, stress or both in the through-the-thickness direction. Such induced strain could cause a piston motion of a mounted mirror or one of many other deformation effects. Without these off-diagonal terms such behavior would not be predicted.

In order to generate these off-diagonal coefficients additional load cases must be performed on the test case model. In a second load case each node on the top and bottom surfaces was given an enforced radial displacement equal to its radial location while the vertical displacements of the same nodes were constrained. Such a deformation generates unit strains in the radial and hoop directions but prevents overall strain in the through-the-thickness direction. The net vertical force acting on the top surface, $F_{3-12}$, was found. Notice, however, that this force is due to unit strains simultaneously enforced in the "1" and "2" directions. We are unable to compute the forces, $F_{31}$ and $F_{32}$, separately because a unit strain in the "1" direction cannot be enforced without generating a unit strain in the "2" direction at the same time. Therefore, in order to compute the individual force contributions, $F_{31}$ and $F_{32}$, it is assumed that,

$$
F_{31}=F_{32}=\frac{F_{3-12}}{2} .
$$

The force, $F_{31}$, defined in Eqn. (10) is then used in Eqn. (8) to generate the correction factor, $k_{31}$. Since $F_{31}$ and $F_{32}$ are equal it follows that $k_{31}$ and $k_{32}$ are also equal. Since the matrix must be symmetric the products $k_{13} k_{11}$ and $k_{23} k_{22}$ in Eqn. (7) can be replaced by $k_{31} k_{33}$ and $k_{32} k_{33}$, respectively. Notice that since we cannot enforce strains in the in-plane directions independently, it is difficult to obtain the correction factors, $k_{11}, k_{22}, k_{12}$, and $k_{21}$. Fortunately, these elements are not significant to proper representation of the effective properties for "hockey puck" bonds which are small compared to the structures they mount. Therefore, we arbitrarily set the constants $k_{11}, k_{22}, k_{12}$, and $k_{21}$ to unity. Equation (7) for use with "hockey puck" bond models with one element through the thickness can be rewritten as in Eqn. (11).

$$
\left\{\begin{array}{l}
\sigma_{11} \\
\sigma_{22} \\
\sigma_{33} \\
\tau_{12} \\
\tau_{23} \\
\tau_{31}
\end{array}\right\}=\left[\begin{array}{cccccc}
M & \frac{v M}{(1-v)} & \frac{k_{31} k_{33} v M}{(1-v)} & 0 & 0 & 0 \\
\frac{v M}{(1-v)} & M & \frac{k_{31} k_{33} v M}{(1-v)} & 0 & 0 & 0 \\
\frac{k_{31} k_{33} v M}{(1-v)} & \frac{k_{31} k_{33} v M}{(1-v)} & k_{33} M & 0 & 0 & 0 \\
0 & 0 & 0 & G & 0 & 0 \\
0 & 0 & 0 & 0 & G & 0 \\
0 & 0 & 0 & 0 & 0 & G
\end{array}\right\}\left\{\begin{array}{l}
\varepsilon_{11} \\
\varepsilon_{22} \\
\varepsilon_{33} \\
\gamma_{12} \\
\gamma_{23} \\
\gamma_{31}
\end{array}\right\}
$$

The correction factors, $k_{31}$ and $k_{33}$, will change for various values of Poisson's ratio and $D / t$ ratio. Therefore, in order to obtain the correct effective properties for a specific combination of these parameters, a newly defined test model must be executed. Using the NASTRAN MESH feature in MSC/NASTRAN ${ }^{\mathrm{TM}}$ a spectrum of "hockey puck" bond models were created and exercised for various pairs of Poisson's ratio and $D / t$ ratio. The correction factors, $k_{31}$ and $k_{33}$, are tabulated in Table 1 to allow the reader to interpolate effective properties. 
Table 1: Correction factors for "hockey puck" bonds with various combinations of $D / t$ ratio and Poisson's ratio.

\begin{tabular}{|c|c|c|c|c|c|c|c|c|}
\hline & \multicolumn{2}{|c|}{$v=\mathbf{0 . 4 5}$} & \multicolumn{2}{c|}{$v=\mathbf{0 . 4 9}$} & \multicolumn{2}{c|}{$v=\mathbf{0 . 4 9 9}$} & \multicolumn{2}{c|}{$v=\mathbf{0 . 4 9 9 9}$} \\
\hline $\boldsymbol{D} / \boldsymbol{t}$ Ratio & $\boldsymbol{k}_{\mathbf{3 3}}$ & $\boldsymbol{k}_{\mathbf{3 1}}$ & $\boldsymbol{k}_{\mathbf{3 3}}$ & $\boldsymbol{k}_{\mathbf{3 1}}$ & $\boldsymbol{k}_{\mathbf{3 3}}$ & $\boldsymbol{k}_{31}$ & $\boldsymbol{k}_{\mathbf{3 3}}$ & $\boldsymbol{k}_{31}$ \\
\hline 1 & 0.3069 & 0.1973 & 0.0710 & 0.1918 & 0.0073 & 0.1908 & 0.0007 & 0.1907 \\
\hline 2 & 0.3665 & 0.3862 & 0.0900 & 0.3761 & 0.0095 & 0.3741 & 0.0010 & 0.3739 \\
\hline 5 & 0.5804 & 0.7443 & 0.2014 & 0.7555 & 0.0244 & 0.7604 & 0.0025 & 0.7609 \\
\hline 10 & 0.7624 & 0.8908 & 0.4172 & 0.9141 & 0.0739 & 0.9250 & 0.0080 & 0.9263 \\
\hline 20 & 0.8746 & 0.9507 & 0.6579 & 0.9682 & 0.2198 & 0.9788 & 0.0295 & 0.9803 \\
\hline 50 & 0.9458 & 0.9814 & 0.8505 & 0.9895 & 0.5580 & 0.9953 & 0.1508 & 0.9966 \\
\hline 100 & 0.9700 & 0.9908 & 0.9209 & 0.9950 & 0.7574 & 0.9981 & 0.3797 & 0.9990 \\
\hline 200 & 0.9822 & 0.9954 & 0.9573 & 0.9976 & 0.8715 & 0.9991 & 0.6342 & 0.9997 \\
\hline 500 & 0.9897 & 0.9981 & 0.9794 & 0.9990 & 0.9440 & 0.9997 & 0.8394 & 0.9999 \\
\hline 1000 & 0.9927 & 0.9992 & 0.9869 & 0.9995 & 0.9689 & 0.9998 & 0.9151 & 0.9999 \\
\hline
\end{tabular}

Figures 6 and 7 show plots of $k_{31}$ and $k_{33}$ vs. $D / t$ ratio for the values of Poisson's ratio shown in Table 1 . However, it is advised that values be taken by interpolation from Table 1 rather than graphically from Figures 6 and 7. For non-circular "hockey puck" bonds an effective diameter, $D_{\text {eff, }}$ is suggested by Lindley and can be computed as,

$$
D_{\text {eff }}=\frac{4 A}{C},
$$

where, $A$ and $C$ are the area and perimeter of the bond, respectively. ${ }^{1}$

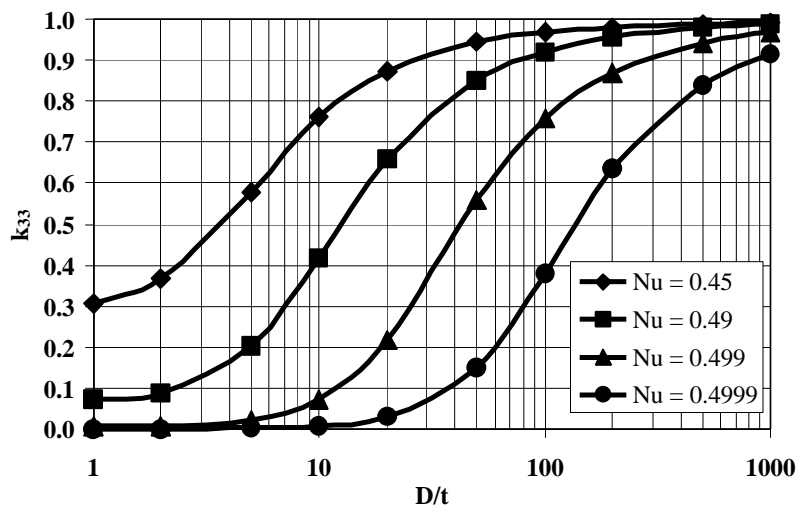

Figure 6: Plots of $k_{33}$ vs. $D / t$ for various values of Poisson's ratio.

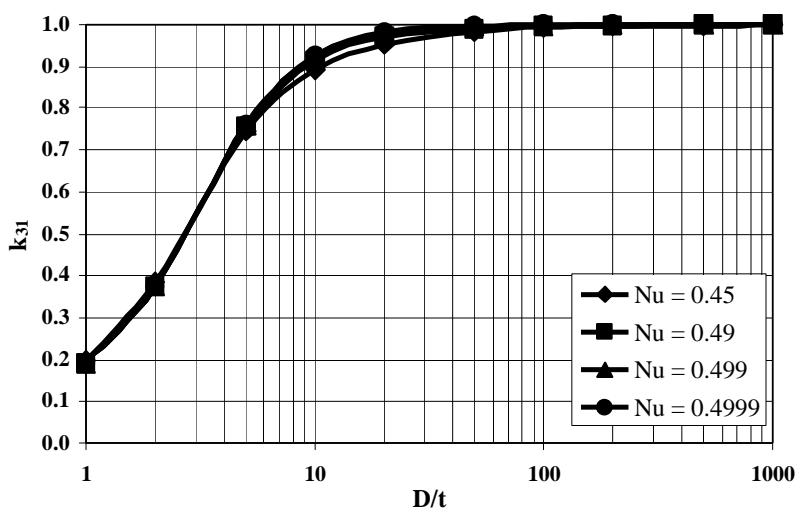

Figure 7: Plots of $k_{3 I}$ vs. $D / t$ for various values of Poisson's ratio.

\subsubsection{Development of effective properties for a ring bond}

Figure 8 shows an example of a ring bond which is to be modeled with one element through the radial direction. The procedure for obtaining effective properties is very similar to that performed for the "hockey puck" bond. However, the form of the Hooke's law matrix is different due to the difference in geometry. In addition, the $b / t$ ratio becomes the geometric parameter analogous to the $D / t$ ratio for "hockey puck" bonds. This Hooke's law is shown in Eqn. (13) as, 


$$
\left\{\begin{array}{l}
\sigma_{11} \\
\sigma_{22} \\
\sigma_{33} \\
\tau_{12} \\
\tau_{23} \\
\tau_{31}
\end{array}\right\}=\left[\begin{array}{cccccc}
k_{11} M & \frac{k_{12} k_{11} v M}{(1-v)} & \frac{k_{13} k_{11} v M}{(1-v)} & 0 & 0 & 0 \\
\frac{k_{12} k_{11} v M}{(1-v)} & k_{11} M & \frac{k_{13} k_{11} v M}{(1-v)} & 0 & 0 & 0 \\
\frac{k_{13} k_{11} v M}{(1-v)} & \frac{k_{13} k_{11} v M}{(1-v)} & k_{33} M & 0 & 0 & 0 \\
0 & 0 & 0 & G & 0 & 0 \\
0 & 0 & 0 & 0 & G & 0 \\
0 & 0 & 0 & 0 & 0 & G
\end{array}\right]\left\{\begin{array}{l}
\varepsilon_{11} \\
\varepsilon_{22} \\
\varepsilon_{33} \\
\gamma_{12} \\
\gamma_{23} \\
\gamma_{31}
\end{array}\right\},
$$

where, the " 1 " direction is radially through the thickness of the ring bond, the " 2 " direction is in the hoop direction, and the " 3 " direction is along the axis of the bond. The correction factors, $k_{12}$ and $k_{13}$, are tabulated in Table 2 for various $b / t$ ratios and Poisson's ratios. It was found that the effective properties for ring bonds are insensitive to the ratio of the radius of the ring bond to its thickness $(R / t)$ for ratios above 10 . Values of $R / t$ less than 10 were not considered. Therefore, these effective properties may also be used for a very long straight bond in which the " 1 " direction is through the thickness of the bond, the " 2 " direction is in the long dimension, and the " 3 " direction is along the width of the bond.

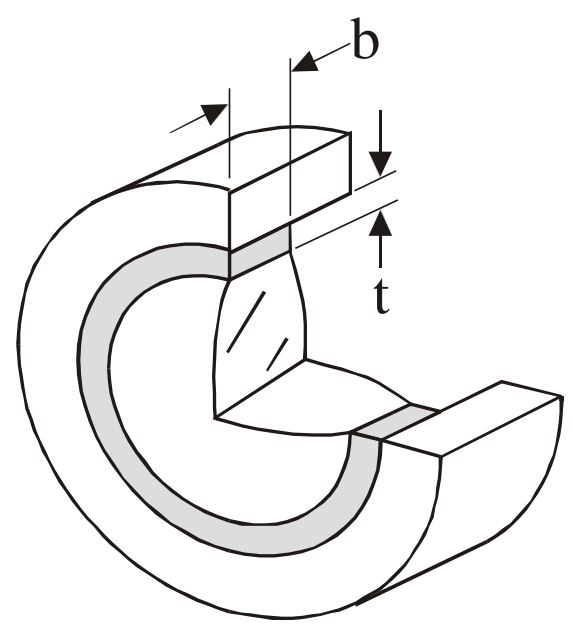

Figure 8: Example of a ring bond design.

Table 2: Correction factors for ring bonds with various combinations of $b / t$ ratio and Poisson's ratio.

\begin{tabular}{|c|c|c|c|c|c|c|c|c|c|c|c|c|c|c|c|c|}
\hline & \multicolumn{4}{|c|}{$v=0.45$} & \multicolumn{4}{|c|}{$v=0.49$} & \multicolumn{4}{|c|}{$v=0.499$} & \multicolumn{4}{|c|}{$v=0.4999$} \\
\hline $\begin{array}{l}b / t \\
\text { Ratio }\end{array}$ & $k_{11}$ & $k_{12}$ & $k_{13}$ & $k_{33}$ & $k_{11}$ & $k_{12}$ & $k_{13}$ & $k_{33}$ & $k_{11}$ & $k_{12}$ & $k_{13}$ & $k_{33}$ & $k_{11}$ & $k_{12}$ & $k_{13}$ & $k_{33}$ \\
\hline 1 & 0.4036 & 0 & 4 & ( & 8 & 9 & 2 & 0.0355 & 0.0108 & 8 & ( & 7 & ( & 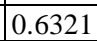 & 0 & 0.0004 \\
\hline 2 & 0.510 & 0.7866 & 257 & 0.3750 & & 7657 & 0.5219 & & & & 13 & & & 07 & 5213 & \\
\hline 5 & .7521 & 0.9267 & 8372 & 0.8518 & 0.36 & 0.9295 & 0.8560 & 3 & 0 & 0. & 30 & 0. & 0. & 19 & 37 & 74 \\
\hline 10 & & 0 & & 0. & & 760 & & & & & 0 & & & & & \\
\hline 20 & 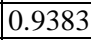 & 0 & 5 & 0. & & 906 & & & & 44 & 0 & & & & 00 & \\
\hline 50 & 0.9756 & 0.9944 & 9876 & 0.9952 & 0.9 & 0.9967 & 0.9933 & 1.0 & 0.7460 & 0.9986 & 0.9973 & 0.9616 & 55 & 0.9991 & 0.9983 & 0.4580 \\
\hline 100 & 0.9883 & 0.9974 & 0.9941 & 0.9904 & 0.9628 & 0.9984 & 0.9968 & 0.9955 & 0.8730 & 0.9994 & 0.9988 & 0.9999 & 0.6005 & 0.9997 & 0.9995 & 0.8299 \\
\hline 200 & 0.9954 & 0.9990 & 0.9977 & 0.9941 & 0.9820 & 0.9993 & 0.9985 & 0.9897 & 0.9365 & 0.9997 & 0.9995 & 0.9994 & 0.7977 & 0.9999 & 0.9998 & 0.9883 \\
\hline 500 & 0.9 & 998 & 96 & & & 0.9998 & 0.9996 & & 0.9 & 0.9999 & 0.9998 & 0.9 & & 1.0000 & 0.9999 & 1.0001 \\
\hline 1000 & 0.9997 & 0.9999 & .9999 & 0.9996 & 0.9985 & 0.9999 & 0.9999 & 0.9985 & 0.9891 & 1.0000 & 0.9999 & 0.9915 & 0.9597 & 1.0000 & 1.0000 & 0.9948 \\
\hline
\end{tabular}

\section{LIMITATIONS}

When employing effective properties in simplified bond models, the user must be aware of their limitation. The effective properties to be used with coarse bond models are simply intended to match the overall stiffness of the bond. Since regions of the bond closer to the free faces will display more compliance, there may be a significant variation in stiffness throughout the bond. Such variation in stiffness may be important to the behavior of the hardware and must, therefore, be included in the model. Use of the effective properties, however, prevents representation of such distribution of stiffness. Therefore, in designs like that shown in Figure 9(a) a detailed model of the bond must be employed.

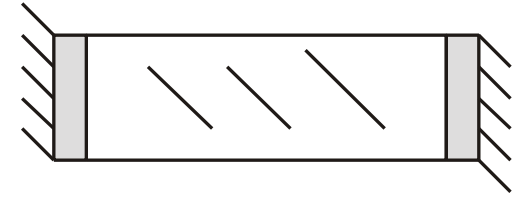

(a)

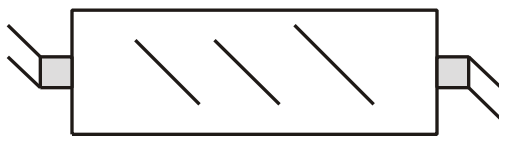

(b)
Figure 9: Cross-section plots of two example ring bond designs: (a) full width bond and (b) partial width bond. 
In order to illustrate this limitation a test case model was developed. A plano-plano circular piece of glass is mounted in a rigid barrel with two ring bond designs as shown in Figures 9(a) and 9(b). Each bond design was modeled with detailed and effective properties. An isothermal soak was applied and the thermoelastic deformation predictions on the front surface of the glass were compared after rigid body surface motions were subtracted. These results are presented in Table 3.

Table 3: Comparison of thermoelastic surface deformations after rigid body motions removed from detailed and effective bond models of two ring bond designs.

\begin{tabular}{|l|l|l|l|l|l|l|}
\cline { 2 - 7 } \multicolumn{1}{c|}{} & \multicolumn{2}{c|}{ Detailed Model } & \multicolumn{2}{c|}{ Effective Model } & \multicolumn{2}{c|}{ \% Error } \\
\hline Design & Surface RMS & Surface P-V & Surface RMS & Surface P-V & Surface RMS & Surface P-V \\
\hline Full Width & $6.21 \mathrm{~nm}$ & $21.57 \mathrm{~nm}$ & $10.06 \mathrm{~nm}$ & $36.46 \mathrm{~nm}$ & $62 \%$ & $69 \%$ \\
\hline Partial Width & $0.18 \mathrm{~nm}$ & $0.70 \mathrm{~nm}$ & $0.17 \mathrm{~nm}$ & $0.65 \mathrm{~nm}$ & $6 \%$ & $7 \%$ \\
\hline
\end{tabular}

Notice that the surface deformation of the full width design is less accurately predicted by the model using effective bond properties compared to the detailed model. However, the correlation in results between the two model types applied to the partial width ring bond design is much better.

Figure 10 shows surface deformations relative to the vertex of the optic. Deformations in Figures 10(a) and 10(b) are shown for the full width bond design for the detailed model and the effective model, respectively. Notice that the optical surface deformation of the effective bond model has greater deformation near the outer edge as compared to the detailed bond model. This increased deformation is due to an excessive stiffness representation of the effective bond model which is local to the free face of the bond. The detailed model on the other hand correctly represents the distribution of stiffness throughout the ring bond including the compliance near its free face. Such stiffness variation cannot be represented by the effective properties, and, therefore, a poor prediction results.

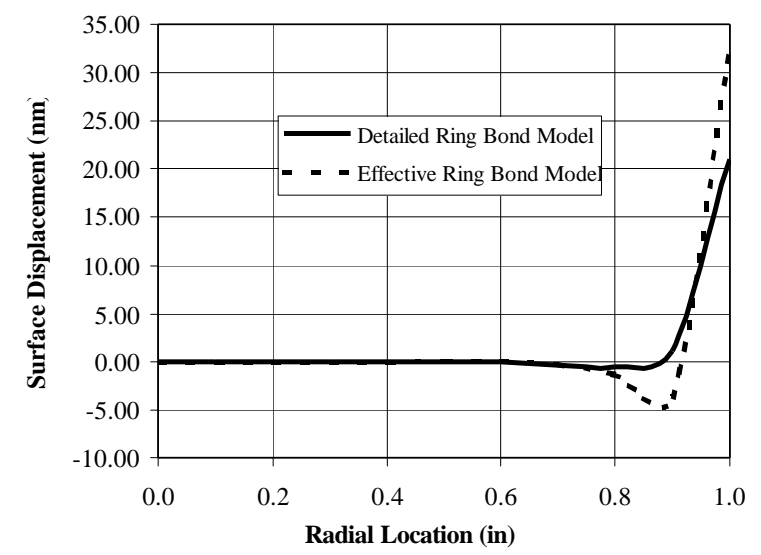

(a)

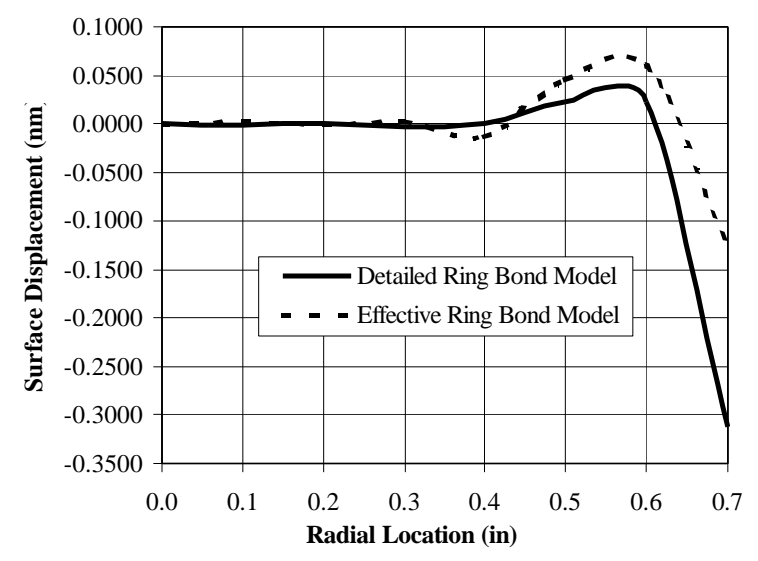

(b)

Figure 10: Comparison of surface deformation predictions by detailed and effective ring bond models for full width bond design shown in Figure 9(a); (a) surface deformation from center to edge of optical surface (1.0 inch radius) and (b) surface deformation from center to 0.7 inch radius sub-aperture. Surface deformations are displacements relative to optical surface vertex and are expressed in nanometers.

In addition, if surface fitting is performed over only a sub-aperture of the full optical surface, then the difference in results is still quite significant. This can be seen in Figure 10(b) in which it is illustrated that the relative difference between the surface deformation predictions between the two model types is significant. Therefore, the analyst should use caution in applying the effective property method to modeling nearly incompressible bonds. 


\section{CONCLUSIONS}

The theory and methods presented above for modeling nearly incompressible bonds with effective properties and a coarse model are a good way to avoid the difficulties commonly associated with modeling these types of components. Furthermore, the reader may develop his or her own test case models in the same manner as was described above in order to develop effective properties for other bond designs. However, since the effective properties simply represent the averaged stiffness of the bond and do not represent the variation of stiffness through the bond, this method may not be applicable for all designs.

\section{REFERENCES}

1. Lindley, P.B., Engineering Design with Natural Rubber, Natural Rubber Technical Bulletin, 3rd Edition, published by the National Rubber Producers Research Association, 1970.

2. Tsai, Hsiang-Chuan and Chung-Chi Lee, "Compressive Stiffness of Elastic Layers Bonded between Rigid Plates," Int. J. Solids Structures Vol.35, No.23, pp.3053-3064,1998.

3. Genberg, V. L., Structural Analysis of Optics, SPIE Short Course, 1986. 